Support information for:

\title{
3D Porous Spherical Sulfur/Carbon Cathode Materials with in Situ Vapor-Phase Polymerized Polypyrrole Coating Layer for High Performance Lithium-Sulfur Batteries
}

Xiuzhen Wang, ${ }^{\dagger},{ }^{\dagger}$ Shuo Zhang, ${ }^{\dagger}$ Haiyang Zhang, ${ }^{\dagger}$ Shan Gao, ${ }^{\dagger}$ Shaojie Han, ${ }^{\dagger}$ Qingyu $\mathrm{Xu},{ }^{\dagger} \AA^{*}$ Jingjing Xu, ${ }^{\dagger *}$ Wei Lu, ${ }^{\dagger^{*}}$ Xiaodong $\mathrm{Wu}^{\dagger}$, Liwei Chen ${ }^{\dagger}$

${ }^{\dagger} i$-LAB, Suzhou Institute of Nano-Tech and Nano-Bionics (SINANO), Chinese Academy of Sciences, Suzhou 215123, Jiangsu, P.R. China.

${ }^{\ddagger}$ Department of Physics, Southeast University, 2 Southeast University Road, Jiangning District, Nanjing 211189, China.

${ }^{\S}$ National Laboratory of Solid State Microstructures, Nanjing University, 22 Hankou Road, Gulou District, Nanjing 210093, China.

Corresponding author:

Qingyu Xu: *E-mail: xuqingyu @ seu.edu.cn;

Jingjing Xu: *E-mail: jjxu2011@ @inano.ac.cn; Tel: (+86)-512-62872503

Wei Lu:*E-mail: wlu2008@ sinano.ac.cn;

The total number of pages is 7 .

The total number of figures is 6 .

There are no tables. 


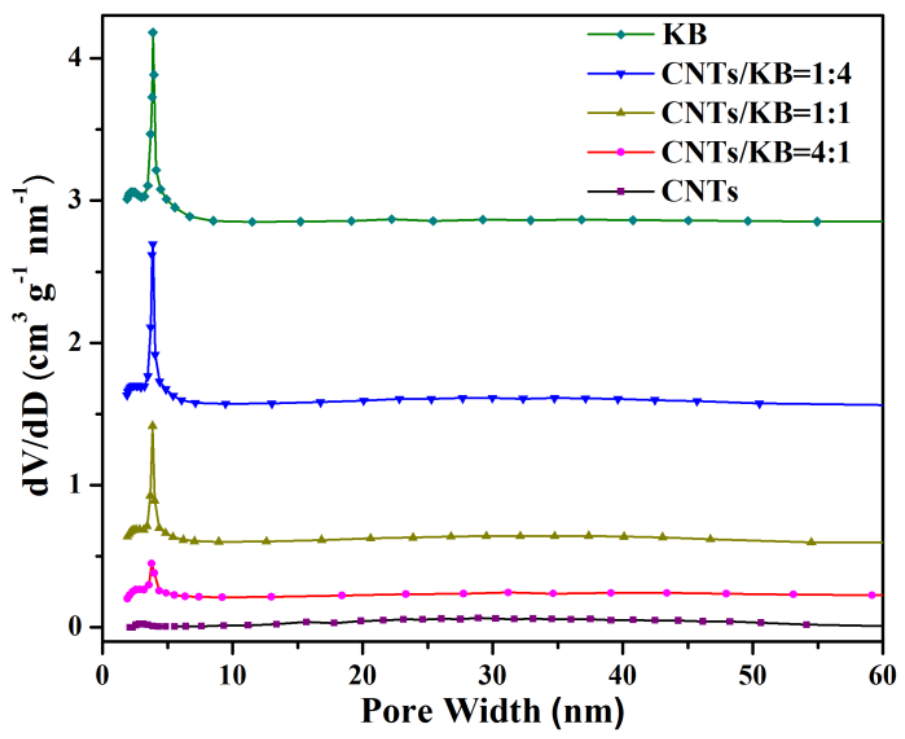

Figure S1. The pore size distributions of the five PSKC microspheres with different $\mathrm{CNT} / \mathrm{KB}$ ratio. 


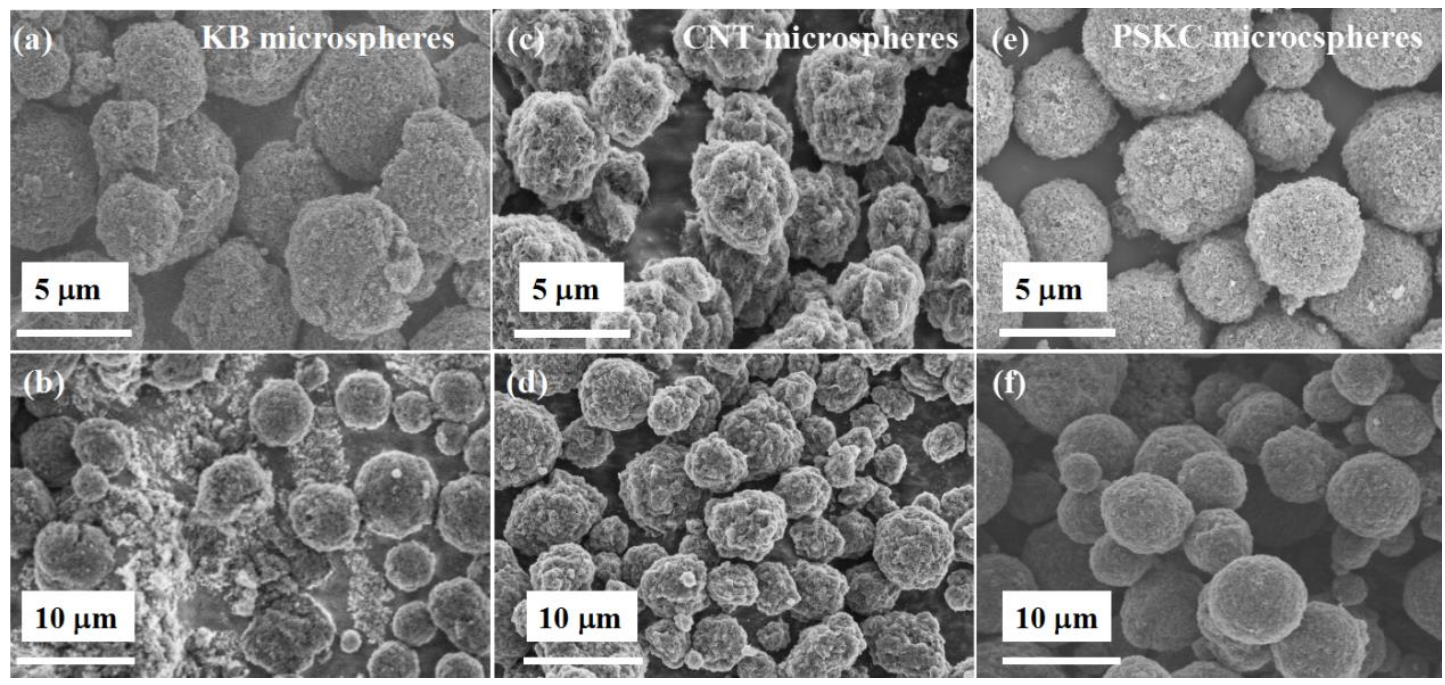

Figure S2. SEM images of pure KB microspheres (a, b), pure CNT microspheres (c, d), and PSKC hybrid microspheres (e, f). 


\section{S/PSKC S/PSKC@PPy}

Figure S3. The photographs of the separators from the cycled cells. 


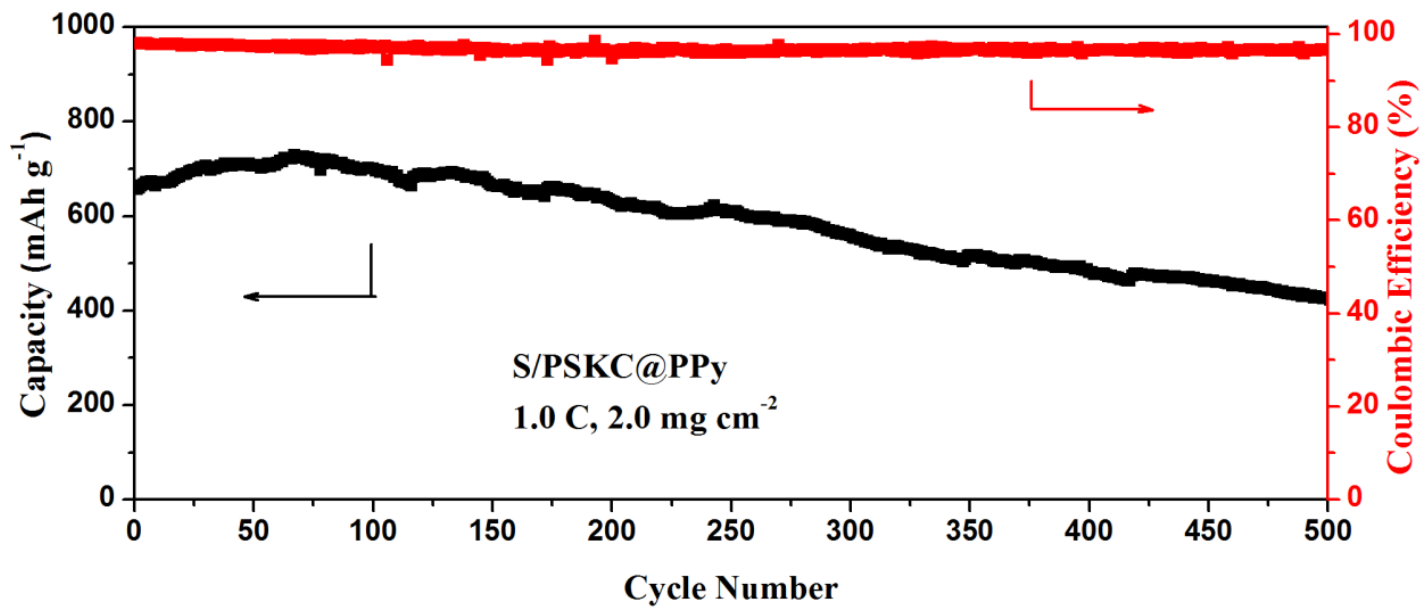

Figure S4. Cyclic performance at $1 \mathrm{C}$ of the S/PSKC@PPy composite with $\mathrm{S}$ loading of $2.0 \mathrm{mg} \mathrm{cm}^{-2}$. 


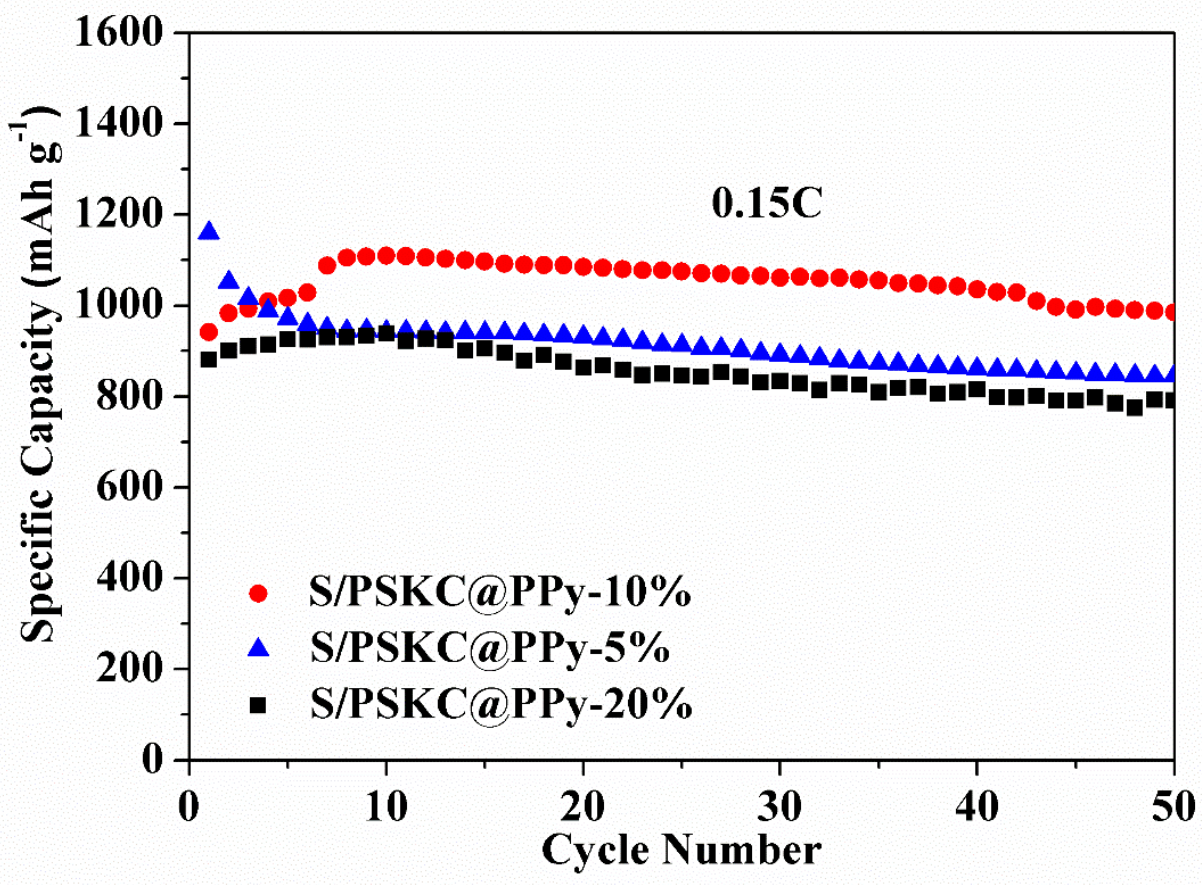

Figure S5. The cycling performance of the S/PSKC@PPy cathodes with different amounts of PPy. 

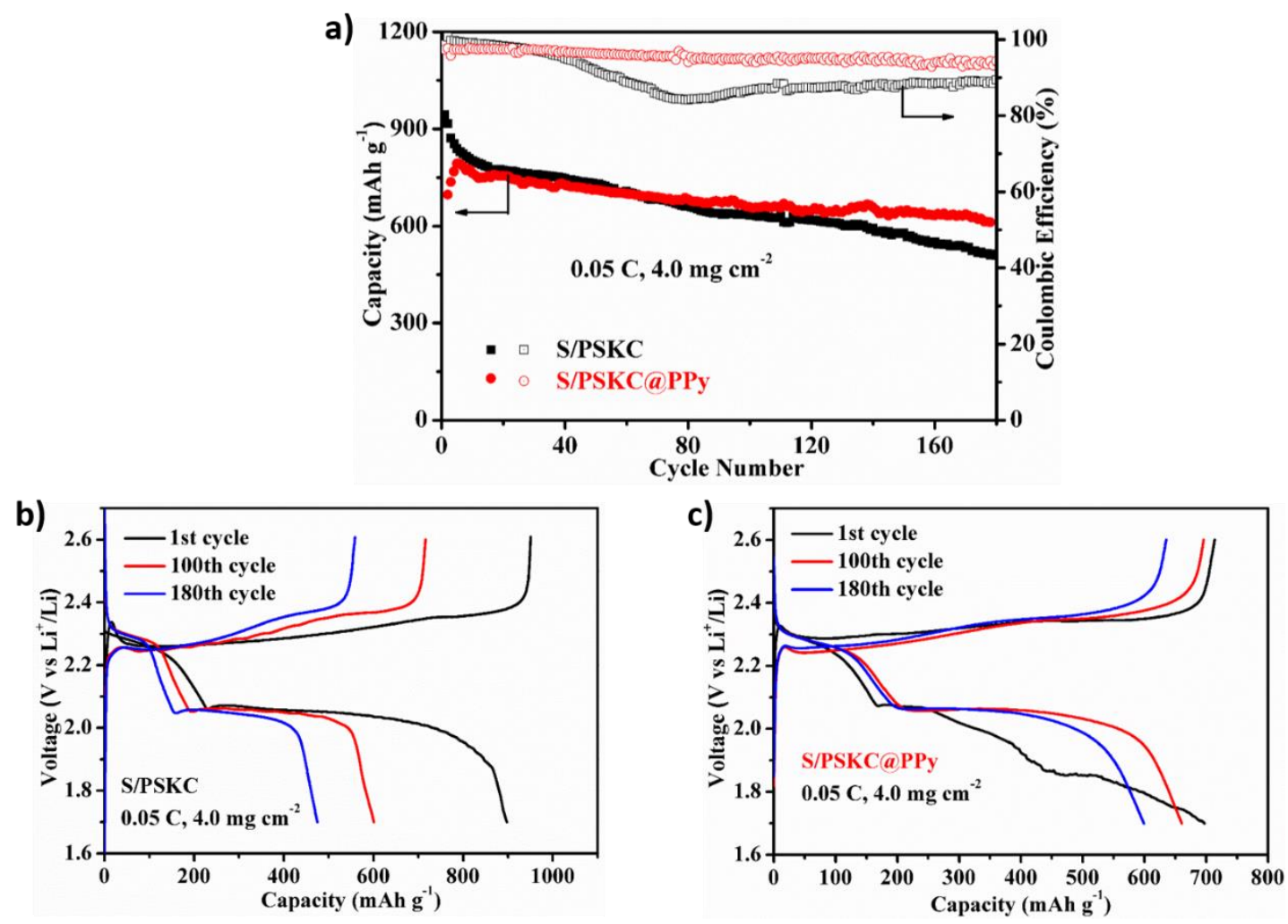

Figure S6. The cycling performance and coulombic efficiency of the S/PSKC and the S/PSKC@PPy with a high sulfur loading of $4.0 \mathrm{mg} \mathrm{cm}^{-2}$ (a). Charge-discharge curves during cycling of the S/PSKC cathode (b) and the S/PSKC@PPy cathode (c). 